\title{
Erythromelanosis Follicularis Faciei: A Case Report and Review of the Literature
}

\author{
Khalid Al Hawsawi $^{\mathrm{a}}$ Ohood Aljuhani $^{\mathrm{a}}$ Ghassan Niaz $^{\mathrm{b}}$ Haneen Fallatah $^{\mathrm{a}}$ \\ Abrar Alhawsawi ${ }^{\mathrm{C}}$ \\ ${ }^{a}$ King Abdul Aziz Hospital, ${ }^{b}$ Umm Alqura University, Makkah, and ${ }^{c}$ King Abdulaziz \\ University, Jeddah, Saudi Arabia
}

\section{Key Words}

Erythromelanosis follicularis faciei · Erythromelanosis follicularis faciei et colli $\cdot$ Keratosis pilaris

\begin{abstract}
Erythromelanosis follicularis faciei is a rare sporadic condition of unknown etiology characterized by reddish-brownish patches and follicular papules that appear commonly on the face and rarely on the neck. Herein, we report a 16-year-old male who had asymptomatic facial skin lesions since early childhood. His family history revealed a similar case in his younger brother. His parents are not consanguineous. Skin examination revealed diffuse nonscaly brownish patches with erythematous background and multiple skin-colored, hypopigmented follicular papules on both cheeks. A summary of previous reports of erythromelanosis follicularis faciei in the literature is presented in this report. $02015 \mathrm{~S}$. Karger AG, Basel
\end{abstract}

\section{Introduction}

Erythromelanosis follicularis faciei (EFF) is a rare sporadic condition of unknown etiology characterized by erythematous hyperpigmented patches and follicular papules on the face. It was first described in Japanese patients in 1960 by Kitamura and collaborators. When the neck is affected, the condition is called erythromelanosis follicularis faciei et colli (EFFC) [1]. The pathogenesis is unknown. However, a combination of vasodilation and hyperpigmentation has been found in the affected areas. Some authors consider EFF as part of the spectrum of keratosis pilaris atrophicans disorders [2]. EFF is characterized clinically by the 
Al Hawsawi et al.: Erythromelanosis Follicularis Faciei: A Case Report and Review of the Literature

presence of red-brown patches on the lateral aspects of the cheeks, and rarely lateral aspects of the neck. Numerous pinhead-sized follicular papules are present within the involved areas that may sometimes appear relatively hypopigmented. Bilateral distribution is the main characteristic, but unilateral cases have been described [1-15]. EFF is usually asymptomatic. However, a burning sensation has been described in a few patients [16-21]. Keratosis pilaris elsewhere in the body is a common association with EFF. Histopathologically, there are hyperkeratosis, slight follicular hyperkeratosis (follicular plugging), increased basal layer pigmentation, dilatation of superficial dermal blood vessels, and periadnexal lymphocytic infiltrate [9].

\section{Case Report}

A 16-year-old male presented with asymptomatic facial skin lesions which he had since early childhood. There was no history of predisposing factors. He had been using topical treatment but without any help. Family history revealed a similar case in his younger brother. His parents are not consanguineous. Skin examination revealed diffuse nonscaly reddishbrownish patches and multiple skin-colored, hypopigmented follicular papules on both cheeks (fig. 1). On the basis of the above classical clinical findings, the diagnosis of EFF was made. The patient was reassured and put under periodic follow-up.

\section{Discussion}

Erythromelanosis follicularis faciei (EFF) is a pigmentary disease associated with erythema and follicular papules on the face. It affects all races. However, it shows a preponderance in the people of Asian ancestry [10-20]. The cause is unknown, but the hereditary component (autosomal recessive) seems to play a role in the pathogenesis [12, 19, 22, 24]. Table 1 summarizes the previous reports of erythromelanosis follicularis faciei (EFF) in the literature.

EFF primarily affects adolescents. However, it has been reported in children as young as 2 years old and in adults as old as 46 years old. Similarly, the onset of the disease shows a wide range, starting from birth to as old as 43 years old. The male:female ratio is 2:1 [1-24]. Differential diagnoses include keratosis pilaris rubra, poikiloderma of Civatte, Riehl's melanosis, and pigmented peribuccal erythrosis of Brocq. In skin type I patients, there may be only erythema, leading to a significant overlap with keratosis pilaris rubra, and it remains to be answered whether EFFC and keratosis pilaris rubra are two spectrums of the same condition [2, 9-18]. Poikiloderma of Civatte is observed in middle-aged women as reticulated dyschromia with atrophy and erythema affecting preferably photoexposed areas and sparing the submental region [5-15]. Treatment is not well defined. Various modalities have been described. Topical agents have been used, including, ammonium lactate, retinoids, hydroquinone, vitamin C, salicylic acid peels (20-30\%), glycolic acid peels, tacalcitol ointment, and metronidazole gel. The evidence for their use is anecdotal. Limited courses of isotretinoin $(0.1-1 \mathrm{mg} / \mathrm{kg} /$ day) have been tried in severe cases. A combination of laser treatment (pulsed dye laser) for erythema and Q-switched Nd:YAG laser for hyperpigmentation have been tried but they require multiple sessions [1-24]. 


\section{Case Reports in Dermatology}

\begin{tabular}{l|l}
\hline \multicolumn{2}{l}{ Case Rep Dermatol 2015;7:335-339 } \\
\hline DOI: $10.1159 / 000442343$ & $\begin{array}{l}\text { C } 2015 \text { S. Karger AG, Basel } \\
\text { www.karger.com/cde }\end{array}$ \\
\hline
\end{tabular}

Al Hawsawi et al.: Erythromelanosis Follicularis Faciei: A Case Report and Review of the Literature

\section{Statement of Ethics}

Consent has been obtained from the parents of the patient for the purpose of using patient's photographs for print or online publication.

\section{Disclosure Statement}

The authors have no conflicts of interest that are directly relevant to the content of this paper. No sources of funding were used to assist in preparation of this paper.

\section{References}

$>2$

Dermatol Ges 2015;13:51-54.

Silva RS, Fonseca JC, Obadia

4 Augustine M, Jayaseelan E: Erythromelanosis follicularis faciei et colli: relationship with keratosis pilaris. Indian J Dermatol Venereol Leprol 2008;74:47-49.

-5 Ertam I, Unal I, Alper S: Erythromelanosis follicularis faciei et colli: report of involvement in two female patients. Dermatol Online J 2005;11:23.

-6 Aljabre SH, Alamir AM, Habiballah NA: Erythromelanosis follicularis faciei: first case report from Saudi Arabia. Ann Saudi Med 2003;23:397-398.

7 Karakatsanis G, Patsatsi A, Kastoridou C, Chaidemenos G, Sotiriadis D: Erythromelanosis follicularis faciei et colli: case reports of bilateral lesions in 2 females. Cutis 2007;79:459-461.

-8 Kurita M, Momosawa A, Ozaki M, Ban I, Harii K: Long-pulsed dye laser for the treatment of erythromelanosis follicularis faciei: report of two clinical cases. Dermatol Surg 2006;32:1414-1417.

-9 Kim MG, Hong SJ, Son SJ, Song HJ, Kim IH, Oh CH, Moon JS: Quantitative histopathologic findings of erythromelanosis follicularis faciei et colli. J Cutan Pathol 2001;28:160-164.

$>10$

Ermertcan AT, Oztürkcan S, Sahin MT, Türkdogan P, Saçar T: Erythromelanosis follicularis faciei et colli associated with keratosis pilaris in two brothers. Pediatr Dermatol 2006;23:31-34. Whittaker SJ, Griffiths WA: Erythromelanosis follicularis faciei et colli. Clin Exp Dermatol 1987;12:33-35. Tüzün Y, Wolf R, Tüzün B, et al: Familial erythromelanosis follicularis and chromosomal instability. J Eur Acad Dermatol Venereol 2000;15:150-152.

13 Lee CW, Yang IS: Cutaneous calcinosis in erythromelanosis follicularis faciei et colli. Clin Exp Dermatol 1987;12:31-32.

14 Warren FM, Davis LS: Erythromelanosis follicularis faciei in women. J Am Acad Dermatol 1995;32:863-866.

15 Mcgills ST, Tuthil RJ, Ratz JL, et al: Unilateral erythromelanosis follicularis faciei et colli in a young girl. J Am Acad Dermatol 1991;25:430-432.

-16 Watt TL, Kaiser JS: Erythromelanosis follicularis faciei et colli. A case report. J Am Acad Dermatol 1981;5:533-534

17 Wang B, Wu Y, Zhu X, Xu XG, Chen HD, Li YH: An exploratory split-face study of a dual-wavelength laser system on erythromelanosis follicularis faciei in Chinese population. J Eur Acad Dermatol Venereol 2014;28:1286-1291.

18 Kim WJ, Song M, Ko HC, Kim BS, Kim MB: Topical tacalcitol ointment can be a good therapeutic choice in erythromelanosis follicularis faciei et colli. J Am Acad Dermatol 2012;67:320-321.

-19 Lalit G, Anubhav G, Kumar KA, Asit M: Familial erythromelanosis follicularis faciei et colli with extensive keratosis pilaris. Int J Dermatol 2011;50:1400-1401.

20 Li YH, Zhu X, Chen JZ, Wu Y, Wei HC, Gao XH, Chen HD: Treatment of erythromelanosis follicularis faciei et colli using a dual-wavelength laser system: a split-face treatment. Dermatol Surg 2010;36:1344-1347.

-21 Sodaify M, Baghestani S, Handjani F, et al: Erythromelanosis follicularis faciei et colli. Int J Dermatol 1994;33:643-644.

-22 Acay MC: Erythromelanosis follicularis faciei et colli: a genetic disorder? Int J Dermatol 1993;32:542.

-23 Sardana K, Relhan V, Garg V, Khurana N: An observational analysis of erythromelanosis follicularis faciei et colli. Clin Exp Dermatol 2008;33:333-336.

24 Yanez S, Velasco JA, Gonzalez MP: Familial erythromelanosis follicularis faciei et colli - an autosomal recessive mode of inheritance. Clin Exp Dermatol 1993;18:283-285. 


\section{Case Reports in Dermatology}

\begin{tabular}{l|l}
\hline \multicolumn{2}{l|}{ Case Rep Dermatol 2015;7:335-339 } \\
\hline DOI: $10.1159 / 000442343$ & $\begin{array}{l}\text { C } 2015 \text { S. Karger AG, Basel } \\
\text { www.karger.com/cde }\end{array}$ \\
\hline
\end{tabular}

Al Hawsawi et al.: Erythromelanosis Follicularis Faciei: A Case Report and Review of the Literature

Table 1. Summary of previous reports of EFF in the literature

\begin{tabular}{|c|c|c|c|c|c|c|c|c|c|}
\hline $\begin{array}{l}\text { First author } \\
\text { [Ref.] }\end{array}$ & $\begin{array}{l}\text { Cases, } \\
\mathrm{n}\end{array}$ & $\begin{array}{l}\text { Patient age, } \\
\text { years }\end{array}$ & Gender & $\begin{array}{l}\text { Patient age at } \\
\text { disease onset }\end{array}$ & $\begin{array}{l}\text { EFFC cases } \\
\text { in the family, } n\end{array}$ & Site of lesion & Keratosis pilaris & $\begin{array}{l}\text { Other associated } \\
\text { conditions }\end{array}$ & Treatment \\
\hline Shahshahani [1] & 60 & $\begin{array}{l}\text { range: } 4-39 \\
\text { average: } 22\end{array}$ & $\begin{array}{l}M=43 \\
F=17\end{array}$ & $\begin{array}{l}\text { at birth, } 3 \text { cases } \\
\text { 1st decade, } 16 \text { cases } \\
\text { 2nd decade, } 33 \text { cases } \\
\text { 4th decade, } 1 \text { case }\end{array}$ & 8 & cheeks and neck & $\begin{array}{l}\text { trunk, arms, } \\
\text { thigh }\end{array}$ & milia in 4 cases & not available \\
\hline \multirow[t]{3}{*}{ Volks [2] } & 3 & 15 & $\begin{array}{l}\text { not } \\
\text { available }\end{array}$ & 4 years & no & cheeks & trunk, limbs & no & no \\
\hline & & $\overline{2}$ & $\mathrm{~F}$ & 1 year & no & cheeks & limbs & no & \\
\hline & & 4 & $\mathrm{~F}$ & birth & 1 (mother) & cheeks, forehead & arms, limbs & no & \\
\hline Silva [3] & 1 & 11 & $\mathrm{M}$ & 3 years & no & face, shoulder, arms & face, neck & no & no \\
\hline \multirow[t]{3}{*}{ Augustine [4] } & 3 & 19 & $\mathrm{~F}$ & 15 years & $\begin{array}{l}2 \text { (sister and } \\
\text { brother) }\end{array}$ & cheeks, neck, ears & $\begin{array}{l}\text { upper back, } \\
\text { shoulders, arms }\end{array}$ & no & no \\
\hline & & $\overline{10}$ & $\mathrm{~F}$ & 6 years & no & cheeks, chin & $\begin{array}{l}\text { upper back, } \\
\text { shoulders, arms }\end{array}$ & no & \\
\hline & & $\overline{13}$ & $\mathrm{M}$ & 8 years & no & cheeks & shoulders & no & \\
\hline \multirow[t]{2}{*}{ Ertam [5] } & 2 & 17 & $\mathrm{~F}$ & 12 years & no & face, neck, arms & arms, upper back, thigh & no & $\begin{array}{l}\text { topical salicylic acid } 2 \% \\
\text { and retinoic acid } 0.01 \%\end{array}$ \\
\hline & & $\overline{19}$ & $\mathrm{~F}$ & 15 years & no & face, neck & arms, upper back, thigh & no & \\
\hline Aljabre [6] & 1 & 17 & $\mathrm{M}$ & 11 years & no & $\begin{array}{l}\text { cheeks, lower lip } \\
\text { and auricles }\end{array}$ & shoulder areas & no & no \\
\hline \multirow[t]{2}{*}{ Karakatsanis [7] } & 2 & 24 & $\mathrm{~F}$ & 23 years & no & cheeks and neck & not available & no & topical retinoids \\
\hline & & $\overline{14}$ & $\mathrm{~F}$ & 10 years & no & cheeks and jaw & not available & no & \\
\hline \multirow[t]{2}{*}{ Kurita [8] } & 2 & 26 & $\mathrm{M}$ & not available & no & cheeks and neck & not available & no & pulsed dye laser \\
\hline & & $\overline{22}$ & $\mathrm{M}$ & not available & no & cheeks & not available & no & \\
\hline Kim [9] & 10 & $\begin{array}{l}\text { range: } 12-46 \\
\text { average: } 22\end{array}$ & $\begin{array}{l}\mathrm{M}=8 \\
\mathrm{~F}=2\end{array}$ & $\begin{array}{l}\text { range: } 8-43 \text { years } \\
\text { average: } 16.5 \text { years }\end{array}$ & no & cheeks and neck & $\begin{array}{l}1 \text { patient with keratosis } \\
\text { pilaris on arms }\end{array}$ & no & not available \\
\hline Ermertcan [10] & 1 & 18 & $\mathrm{M}$ & childhood & 1 (brother) & maxilla, cheeks, neck & arm & no & oral isotretinoin \\
\hline Whittaker [11] & 1 & 15 & $\mathrm{M}$ & 13 years & no & preauricular regions & arms & no & topical retinoic acid \\
\hline Tuzun [12] & 1 & 17 & $\mathrm{M}$ & 10 years & $\begin{array}{l}2 \text { (sister and } \\
\text { father) }\end{array}$ & cheeks & arms and trunk & $\begin{array}{l}\text { diabetes mellitus } \\
\text { and congenital } \\
\text { leukokeratosis }\end{array}$ & no \\
\hline Lee [13] & 1 & 18 & $\mathrm{M}$ & 13 years & no & cheeks, neck & no & no & no \\
\hline \multirow[t]{2}{*}{ Warren [14] } & 2 & 35 & $\mathrm{~F}$ & 25 years & no & cheeks & arms & no & $\begin{array}{l}\text { topical ammonium lactate } \\
12 \% \text { or metronidazole gel }\end{array}$ \\
\hline & & $\overline{43}$ & $\mathrm{~F}$ & $241 / 2$ years & no & & & no & \\
\hline McGillis [15] & 1 & 13 & $\mathrm{~F}$ & 8 years & no & face and neck & upper arms & no & $\begin{array}{l}\text { topical tretinoin } 0.05 \% \text {, } \\
\text { ammonium lactate and } \\
\text { hydroquinone }\end{array}$ \\
\hline$\overline{\text { Watt [16] }}$ & 1 & 15 & $\mathrm{M}$ & several months & no & $\begin{array}{l}\text { temples, cheeks } \\
\text { and neck }\end{array}$ & upper arms & no & no \\
\hline Wang [17] & 12 & $\begin{array}{l}\text { not } \\
\text { available }\end{array}$ & $\begin{array}{l}F=7 \\
M=5\end{array}$ & not available & $\begin{array}{l}\text { not } \\
\text { available }\end{array}$ & cheeks & not available & not available & $\begin{array}{l}\text { dual wavelength laser } \\
\text { (pulsed dye laser + } \\
\text { Q-switched Nd:YAG laser }\end{array}$ \\
\hline Kim [18] & 11 & $14.4 \pm 7.7$ & $\begin{array}{l}M=6 \\
F=5\end{array}$ & not available & $\begin{array}{l}\text { not } \\
\text { vailable }\end{array}$ & $\begin{array}{l}\text { preauricular and } \\
\text { maxillary regions }\end{array}$ & not available & not available & $\begin{array}{l}\text { topical tacalcitol } \\
\text { ointment }\end{array}$ \\
\hline Lalit [19] & 1 & 21 & $\mathrm{M}$ & 19 years & no & cheeks forehead chin & arms, shoulders, back & no & no \\
\hline Li [20] & 1 & 20 & $\mathrm{M}$ & 12 years & no & $\begin{array}{l}\text { preauricular area, } \\
\text { neck }\end{array}$ & $\begin{array}{l}\text { shoulders, lateral arms, } \\
\text { thighs }\end{array}$ & no & $\begin{array}{l}\text { dual-wavelength laser } \\
\text { system (pulsed dye laser + } \\
\text { Q-switched Nd:YAG laser }\end{array}$ \\
\hline Sodaify [21] & 3 & $15,18,28$ & $\mathrm{M}$ & childhood & no & cheeks & arms and legs & no & $\begin{array}{l}\text { tretinoin and hydroquinone } \\
\text { cream }\end{array}$ \\
\hline \multirow[t]{2}{*}{ Acay [22] } & 2 & 17 & $\mathrm{M}$ & 9 years & 1 (sister) & cheeks and neck & upper arms & no & not available \\
\hline & & $\overline{17}$ & $\mathrm{M}$ & childhood & $\begin{array}{l}2 \text { (mother and } \\
\text { grandmother) }\end{array}$ & cheeks & face, neck & no & \\
\hline \multirow[t]{5}{*}{ Sardana [23] } & 5 & 19 & $\mathrm{M}$ & 13 years & no & temples, cheeks, neck & arms, shoulders, back & not available & oral isotretinoin \\
\hline & & $\overline{13}$ & $\mathrm{M}$ & not available & no & & & not available & topical retinoic acid cream \\
\hline & & $\overline{18}$ & $\mathrm{M}$ & 12 years & no & & & not available & $\begin{array}{l}\text { tretinoin and hydroquinone } \\
\text { cream }\end{array}$ \\
\hline & & $\overline{11}$ & $\mathrm{M}$ & 5 years & no & & & not available & \\
\hline & & $\overline{13}$ & $\mathrm{~F}$ & not available & no & & & not available & \\
\hline \multirow[t]{2}{*}{ Yanez [24] } & 2 & 15 & $\mathrm{M}$ & 10 years & 1 (sister) & $\begin{array}{l}\text { cheeks, forehead } \\
\text { and neck }\end{array}$ & arms & no & $\begin{array}{l}\text { topical retinoic acid cream } \\
0.05 \%\end{array}$ \\
\hline & & $\overline{18}$ & $\mathrm{~F}$ & childhood & 1 (brother) & cheeks & upper limbs & no & \\
\hline $\begin{array}{l}\text { Al Hawsawi, } \\
\text { this study }\end{array}$ & 1 & 16 & $\mathrm{M}$ & childhood & 1 (brother) & cheeks & no & no & no \\
\hline
\end{tabular}


Al Hawsawi et al.: Erythromelanosis Follicularis Faciei: A Case Report and Review of the Literature

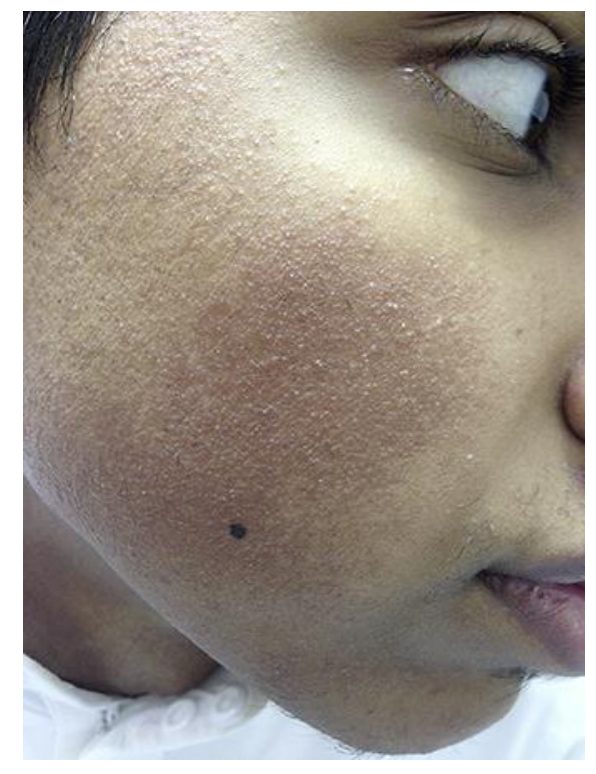

Fig. 1. Diffuse nonscaly reddish-brownish patches with multiple skin-colored, hypopigmented follicular papules on both cheeks. 